\title{
Adiestramiento en el diagnóstico de las parasitosis intestinales en la red de laboratorios de Cuba
}

\author{
Training for diagnosis of intestinal parasites \\ in the national laboratory system in Cuba
}

Fidel Angel Núñez 1

Carlos M. Finlay 1

\footnotetext{
1 Instituto de Medicina Tropical "Pedro Kourí". Apartado Postal 601, Marianao 13, Ciudad Habana, Cuba. fan@ipk.sld.cu
}

\begin{abstract}
A national training project in the diagnosis of intestinal parasites was conducted in 1997. An initial national course was followed respectively by courses in the Central, Eastern, and Western Provinces. Our results showed that Cryptosporidium parvum, Cyclospora cayetanensis, and leukocytes showed a significantly lower percentage of errors after the training than before $(p<0.01)$. The same occurred with Entamoeba histolytica/E. dispar and Chilomastix mesnilii $(p<0.05)$. Among the helminths, Taenia spp., Fasciola hepatica, and hookworm showed significantly fewer errors after the training $(p<0.01)$. In the other specimens, few mistakes were found both at the beginning and after the training, and the percentage of errors did not change ( $p>0.05)$. Furthermore, when comparing scores before and after training, a significant increase in median scores appeared in the Central Provinces $(p<0.05)$, Western Provinces $(p<0.01)$, and in the entire series $(p<0.01)$. The results showed the effectiveness of this intervention; these periodic mandatory training courses should be developed together with national programs for quality assessment in Parasitology.
\end{abstract}

Key words Quality Control; Parasites; Parasitology

Resumen En 1997, se desarrolló un proyecto nacional de adiestramiento integral para el diagnóstico de las parasitosis intestinales, el que incluyó 4 cursos; uno com carácter nacional, y otros 3 en las provincias centrales, orientales y occidentales. Al comparar los errores diagnósticos, vimos que Cryptosporidium parvum, Cyclospora cayetanensis y los leucocitos mostraron un porcentaje significativamente menor de fallas después del curso que al comienzo $(p<0,01)$, al igual que con Entamoeba histolytica/E. dispar y Chilomastix mesnilii $(p<0,05)$. Entre los helmintos, Taenia $s p$., Fasciola hepatica y los ancylostomídeos redujeron significativamente el número de fallas $(p<0,01)$. En el resto de las especies se encontraron muy pocos errores, y los porcentajes de fallas no variaron al finalizar los cursos $(p>0,05)$. Por otra parte, al comparar las medias de las puntuaciones antes y después de los cursos, se encontró un aumento significativo en el curso de las provincias centrales $(p<0,05)$, en las occidentales $(p<0,01)$, y en la totalidad $(p<0,01)$, lo que demonstró la efectividad a corto plazo de esta intervención. Sin embargo, pensamos que estas actividades de educación continuada deberian seguirse junto com programas nacionales para el control de la calidad.

Palabras-clave Control de Calidad; Parasitos; Parasitología 


\section{Introducción}

Las infecciones intestinales por helmintos y protozoos están entre las más comunes del hombre a nivel mundial, pues se encuentran distribuidas en todas las regiones tropicales y templadas del planeta; sin embargo, el ser más prevalentes en los países en desarrollo y en las comunidades más pobres ha traído como consecuencia la tendencia a considerar estas infecciones sólo como un producto de las condiciones de vida, y que sean subestimadas por los servicios de salud pública (Savioli et al., 1992; Chan, 1997). A pesar de esto, algunas parasitosis intestinales como la cryptosporidiosis han cobrado importancia en países desarrollados; por ejemplo, en los Estados Unidos, esta coccidia fue responsable, en 1993, de un brote de transmisión hídrica en Milwaukee, Wisconsin, que involucró a 403 mil personas (Griffiths, 1998); otro protozoo intestinal, Giardia lamblia, está siendo considerado la causa principal de brotes de transmisión hídrica (Marshall et al., 1997) y es responsable de un mínimo de 4.000 admisiones hospitalarias al año en ese país (WHO, 1992).

En Cuba, el parasitismo intestinal más prevalente ha sido el causado por Trichuris trichiura (Núñez et al., 1993), del que se reportan mayores cifras de prevalencia en zonas rurales y montañosas (Núñez et al., 1993; Ramírez \& Dona, 1993). Otros grupos de riesgo son los niños que asisten a guarderías infantiles, los que se han encontrado infectados con tasas altas de prevalencia por G. lamblia (Núñez et al., 1999) y Enterobius vermicularis (Núñez et al., 1996). En los últimos años, algunas parasitosis intestinales "emergentes" como Cryptosporidium parvum y Cyclospora cayetanensis han ido ganando importancia tanto en pacientes inmunocompetentes, como en enfermos de SIDA (Hadad et al., 1993; Núñez et al., 1995; Suarez et al., 1997; Escobedo \& Núñez, 1999).

En la red nacional de laboratorios de Ciudad Habana se realizó, en 1993, un estudio sobre la calidad del diagnóstico de las parasitosis intestinales, en el que se demostraron deficiencias en el diagnóstico de protozoos y helmintos intestinales (Núñez et al., 1997). Teniendo en cuenta estos resultados, se desarrolló, en 1994, una intervención educativa en la red de salud de la ciudad, la que produjo una mejoría importante del diagnóstico en los centros que participaron en los adiestramientos (Núñez et al., 1998); posteriormente, se han desarrollado otros estudios aislados sobre la calidad del diagnóstico parasitológico en nuestro país (Laird et al., 1996). Teniendo en cuenta estos anteceden- tes y la necesidad de desarrollar acciones educativas para mejorar el diagnóstico parasitológico en la red nacional de laboratorios, se elaboró, en 1997, un proyecto de adiestramiento integral que incluyó a las 14 provincias del país y al municipio especial, Isla de la Juventud. El objetivo de este trabajo es explicar los principales errores diagnósticos encontrados en la red nacional de laboratorios y el comportamiento de las evaluaciones en los asistentes a los cursos desarrollados en este proyecto nacional de adiestramiento integral para el diagnóstico de las parasitosis intestinales.

\section{Material y métodos}

\section{Diseño de la intervención}

Para nuestra intervención desarrollamos varios cursos que involucraron a toda la red nacional de laboratorios de salud pública, y para su organización se tuvo en cuenta la estructura de la división político administrativa de nuestro país, en el que existen 14 laboratorios de Parasitología provinciales; en cada provincia este laboratorio se encuentra en el Centro Provincial de Higiene, Epidemiología y Microbiología (CPHEM), que es la máxima instancia organizativa y normativa en la red provincial de laboratorios que realizan diagnóstico parasitológico y que abarcan a todos los Centros Municipales de Higiene, Epidemiología y Microbiología (CHEM), hospitales y policlínicas del área geográfica. Además se incluyó un laboratorio municipal con importancia similar a los provinciales por ser la cabecera del municipio especial, que fue el laboratorio de Isla de la Juventud. En todos los cursos se impartieron aspectos teóricos y prácticos, donde se profundizó en las habilidades para la identificación de los estadios morfológicos de los diferentes parásitos intestinales y para ello se tuvieron en cuenta las experiencias previas (Núñez et al., 1998).

En total, se impartieron 4 cursos; uno nacional, al que se invitó a todos los médicos parasitólogos que dirigen la actividad en cada una de las 14 provincias y en el municipio especial, y otros 3 cursos sucesivos, uno para los representantes de las provincias centrales, otro para las orientales, y el último para las occidentales. En todos los cursos se entregaron materiales impresos o fotocopiados, como folletos sobre técnicas coproparasitológicas, juegos de viales con muestras positivas preservadas, y láminas positivas con parásitos. Además se realizaron pruebas prácticas tanto al comienzo como al finalizar los mismos, empleando viales plásticos 
con formas parasitarias preservadas en formaldehído al 5\%, en concentración de 4 o más parásitos por campo, visto con aumento de 400 veces, en forma similar a la empleada en controles de calidad anteriores (Núñez et al., 1998).

Por último, se entregó el material de la Organización Mundial de la Salud (OMS) titulado Medios Auxiliares para el Diagnóstico de las Parasitosis Intestinales, que consiste en un conjunto de 9 láminas plastificadas que contienen fotomicrografías de parásitos intestinales y claves para la identificación morfológica de helmintos y protozoos intestinales; además de procederes y marchas técnicas recomendadas (Orihel et al., 1997), para utilizarse como medio de consulta por los 14 laboratorios provinciales del país y por el de la Isla de la Juventud.

\section{Análisis estadístico}

Para comparar los porcentajes de errores diagnósticos en la evaluación realizada tanto al comienzo como al final de los cursos para cada elemento a examinar, se empleó una prueba de comparación de proporciones, considerándose significativo un valor de $\mathrm{p}<0,05$. Para comparar las puntuaciones medias obtenidas tanto al comienzo como al final de cada uno de los cursos y en la totalidad de los mismos, se empleó la prueba no paramétrica de KruskallWallis para la comparación de medias, y se consideró como significativo un valor de $\mathrm{p}<0,05$.

\section{Resultados}

En la Tabla 1, al analizar las fallas en el diagnóstico según los diferentes elementos a identificar, se vio un número significativamente menor de errores después del curso que a su comienzo $(\mathrm{p}<0,01)$ con las coccidias: Cryptosporidium parvum y C. cayetanensis. Lo mismo ocurrió con Entamoeba histolytica/E. dispar $(\mathrm{p}<0,05)$ y con los leucocitos que fueron empleados en un sistema simulado $(p<0,01)$; todos estos elementos fueron los principales errores diagnósticos tanto al comienzo como al final. El protozoo Chilomastix mesnilii también redujo significativamente el número de errores, pero en menor grado $(p<0,05)$.

En los helmintos intestinales, Taenia spp., Fasciola hepatica y los ancylostomídeos, también se redujeron significativamente el número de fallas al comparar los porcentajes de éstas, antes y después del adiestramiento $(\mathrm{p}<0,01)$.

En otros parásitos, como Ascaris lumbricoides, Entamoeba coli, G. lamblia, Hymenolepis nana, E. vermicularis, y Blastocystis hominis, se encontraron muy pocos errores tanto al comienzo como al final de los cursos, y los porcentajes de fallas no sufrieron variación ( $p>0,05)$, mientras que T. trichiura y Endolimax nana fueron los únicos parásitos en los que no se encontraron errores en el diagnóstico, tanto en las evaluaciones del comienzo como en las del final.

En la Tabla 2, se muestra la distribución del tiempo de las clases impartidas en cada uno de los cursos, para un total de 200 horas de clases teóricas y prácticas, y una asistencia de 62 participantes, en su mayoría profesionales de todo el país. Es de destacar que el curso nacional tuvo una duración mayor que los demás, porque en él se capacitaron los especialistas en Parasitología, que replicarían después la actividad en sus respectivas provincias. Además, se reflejan los resultados de la media de la puntuación obtenida por los alumnos de cada uno de los cursos, como se puede apreciar al comparar las medias de las puntuaciones antes y después del curso (prueba de Kruskall-Wallis): se produce un aumento significativo de la puntuación en el curso de las provincias centrales $(\mathrm{p}<0,05)$, el de las occidentales $(p<0,01)$, y en la totalidad de los cursos $(p<0,01)$.

\section{Discusión}

En la identificación de las diferentes especies parasitarias empleadas en las evaluaciones, se observó un número mayor de errores en la evaluación de comienzo, con C. parvum y C. cayetanensis, los que demostraron una mejoría significativa en la evaluación final de los cursos. Esto nos induce a pensar que estas coccidias intestinales son poco diagnosticadas debido a su desconocimiento en los laboratorios de la red nacional, a pesar de que han ido ganando en importancia tanto en individuos inmunocompetentes, como en personas inmucomprometidas (Hadad et al., 1993; Núñez et al., 1995; Suarez et al., 1997; Ortega et al., 1998; Escobedo \& Núñez, 1999). En los Estados Unidos, se han informado falsos brotes por sobrediagnóstico de Cryptosporidium en la ciudad de Nueva York, y por Cyclospora en la Florida, los que han demostrado la necesidad de entrenamientos sobre estos microorganismos emergentes en las redes de laboratorios (Anonymous, 1997); por otra parte, en un estudio realizado a una muestra aleatoria estratificada de los médicos de Connecticut, se concluyó que, probablemente, la cryptosporidiosis no se reconoce o se subdiagnostica en este estado, lo que pudiera estar ocurriendo debido a que muchos médicos desconocen esta parasitosis y sus síntomas 
Errores prácticos en la identificación de elementos parasitarios y leucocitos, al comienzo y al finalizar los cursos. Cuba, 1997.

\begin{tabular}{|c|c|c|c|c|c|c|c|}
\hline \multirow[t]{3}{*}{ Elemento a identificar } & \multirow{2}{*}{\multicolumn{2}{|c|}{$\begin{array}{l}\text { Número de viales usados } \\
\text { en cada evaluación }\end{array}$}} & \multicolumn{4}{|c|}{ Diagnósticos incorrectos } & \multirow[t]{3}{*}{$p^{*}$} \\
\hline & & & \multicolumn{2}{|c|}{ 1ra evaluación } & \multicolumn{2}{|c|}{ 2da evaluación } & \\
\hline & $1 \mathrm{ra}$ & $2 \mathrm{da}$ & $\mathrm{n}$ & $\%$ & $n$ & $\%$ & \\
\hline Cryptosporidium parvum & 15 & 15 & 12 & 80,0 & 4 & 26,6 & $<0,01$ \\
\hline Cyclospora cayetanensis & 25 & 25 & 18 & 72,0 & 3 & 12,0 & $<0,01$ \\
\hline Fasciola hepatica & 40 & 58 & 25 & 62,5 & 6 & 10,3 & $<0,01$ \\
\hline leucocitos & 58 & 43 & 35 & 60,3 & 4 & 9,3 & $<0,01$ \\
\hline Entamoeba histolytica/E. dispar & 43 & 43 & 24 & 55,8 & 13 & 30,2 & $<0,05$ \\
\hline Chilomastix mesnilii & 18 & 18 & 7 & 38,9 & 1 & 5,5 & $<0,01$ \\
\hline Ancylostomídeos & 33 & 18 & 12 & 36,4 & 0 & 0,0 & $<0,01$ \\
\hline Taenia spp. & 58 & 58 & 15 & 25,8 & 1 & 1,7 & $<0,01$ \\
\hline Ascaris lumbricoides & 30 & 30 & 6 & 20,0 & 3 & 10,0 & $>0,05$ \\
\hline Entamoeba coli & 15 & 15 & 2 & 13,3 & 0 & 0,0 & $>0,05$ \\
\hline Giardia lamblia & 33 & 33 & 4 & 12,1 & 0 & 0,0 & $>0,05$ \\
\hline Hymenolepis nana & 18 & 18 & 2 & 11,1 & 1 & 5,5 & $>0,05$ \\
\hline Enterobius vermicularis & 30 & 48 & 3 & 10,0 & 0 & 0,0 & $>0,05$ \\
\hline Blastocystis hominis & 43 & 30 & 3 & 6,9 & 2 & 6,6 & $>0,05$ \\
\hline
\end{tabular}

* Comparación de proporciones.

Tabla 2

Cursos de adiestramiento en Parasitología Intestinal desarrollados en la red nacional de salud pública. Cuba, 1997.

\begin{tabular}{|c|c|c|c|c|c|}
\hline \multirow[t]{2}{*}{ Alcance del curso (Nivel) } & \multirow{2}{*}{$\begin{array}{l}\text { Tiempo de } \\
\text { duración }\end{array}$} & \multirow{2}{*}{$\begin{array}{l}\text { No. de } \\
\text { alumnos }\end{array}$} & \multicolumn{2}{|c|}{ Puntuación alcanzada } & \multirow[t]{2}{*}{$p^{*}$} \\
\hline & & & $\begin{array}{c}\text { Comienzo } \\
\text { Media ( } \pm \text { DE) }\end{array}$ & $\begin{array}{c}\text { Final } \\
\text { Media ( } \pm \text { DE) }\end{array}$ & \\
\hline Nacional & 80 horas & 15 & $8,8( \pm 1,21)$ & $9,9( \pm 0,26)$ & $>0,05$ \\
\hline Provincias centrales & 40 horas & 18 & $7,5( \pm 1,46)$ & $9,4( \pm 0,70)$ & $<0,05$ \\
\hline Provincias orientales & 40 horas & 19 & $5,2( \pm 1,65)$ & $8,2( \pm 2,35)$ & $>0,05$ \\
\hline Provincias occidentales & 40 horas & 10 & $5,3( \pm 1,77)$ & $8,0( \pm 1,05)$ & $<0,01$ \\
\hline Total & 200 horas & 62 & $6,7( \pm 2,11)$ & $8,9( \pm 1,60)$ & $<0,01$ \\
\hline
\end{tabular}

$\mathrm{DE}=$ Desviación estándar

* Prueba de Kruskall-Wallis

y, por lo tanto, no indican los exámenes adecuados para su búsqueda (Morin et al., 1997). En otros países de América Latina, también se han detectado problemas con el diagnóstico de las coccidias (Weitz \& Astorga, 1995).

En cuanto al diagnóstico de leucocitos, se produjo una mejoría importante después del adiestramiento, siendo confundido en casi la totalidad de los casos errados con E. histolyti$c a / E$. dispar, que también presentó un porcentaje alto de fallas diagnósticas, las que mejoraron al final del entrenamiento. Es un hecho conocido, la confusión diagnóstica de este protozoo con leucocitos y macrófagos, lo que puede traer como consecuencia que, frecuentemente, se sobrediagnostique la amebiasis, sobre todo en casos de niños con diarreas provocadas por enterobacterias (Fonte et al., 1998; Haque et al., 1998).

Con respecto al diagnóstico de los helmintos intestinales, los huevos de Taenia sp., F. hepatica, y ancylostomídeos se encontraron con altos porcentajes de errores en su identificación, desde la evaluación inicial, a pesar de producirse una mejoría notable en las evaluaciones finales. Estudios previos han demostrado que F. hepatica y Taenia sp. están entre los parásitos que confrontan mayor números de 
fallas diagnósticas en nuestro medio (Laird et al., 1996; Núñez et al., 1997, 1998); por otra parte, debemos destacar que Fasciola, a pesar de no ser frecuente, puede presentarse en forma de brotes epidémicos en nuestro país (Núñez et al., 1998).

Estos resultados confirman la existencia de importantes deficiencias en el diagnóstico de las parasitosis intestinales en nuestra red nacional de laboratorios. En algunos países como en Rusia, se han demostrado pocos conocimientos de la Parasitología en el personal médico, puesto que la educación que se brinda a los estudiantes en las instituciones de educación superior es escasa y fragmentada, lo que ha motivado, en los últimos años, la creación y modificación de varios programas de postgrado, con el objetivo de fortalecer sus conocimientos teóricos y prácticos (Zueva \& Lysenko, 1997; Iafaev, 1998). De acuerdo con estudios realizados en Europa, en los que se han involucrado diferentes países de la región, como Inglaterra, Francia, Alemania, Italia y Polonia, se ha verificado que el estado actual de la enseñanza de la Parasitología en sus facultades de Medicina es inadecuado (Pawlowski et al., 1998) y que, en ocasiones, la enseñanza emplea métodos anacrónicos (Eyckmans, 1997). Han llegado a la conclusión de que la enseñanza básica de pregrado en Parasitología, en muchas facultades europeas, es deficiente, por lo que se debe aumentar el número de horas obligatorias, tanto en el área preclínica como en las áreas clínicas (Pawlowski et al., 1998). En el área de América Latina, existen pocos estudios publicados sobre el control de la calidad del diagnóstico de las parasitosis (Núñez et al., 1998) y el estado de la enseñanza en Parasitología.
La Parasitología Médica es una disciplina relativamente joven en comparación con la Microbiología tradicional, pero su conocimiento es esencial, a través de un amplio rango de disciplinas biomédicas que se relacionan con problemas frecuentes de la práctica médica, los que van desde la Medicina Tropical hasta las complicaciones fatales en el SIDA y otras enfermedades donde existe compromiso inmunológico (Pawlowski et al., 1998); además, está en constante avance y renovación, por lo que sus contenidos pueden caducar en poco tiempo (Anderson, 1997).

Los resultados obtenidos en esta intervención demuestran que se produjo una mejoría notable en los promedios de las calificaciones en la totalidad de los 62 participantes evaluados en toda la red nacional de laboratorios. Sin embargo, a pesar de los resultados obtenidos en la primera evaluación posterior a los cursos, consideramos que sólo se garantizará un mejoramiento sostenido de la calidad del diagnóstico parasitológico en nuestra red nacional de salud pública, si estas actividades de educación continuada se mantienen en forma periódica, y se establecen programas nacionales para el control de la calidad (Petithory \& Drouhet, 1990; Wood et al., 1994; Laird et al., 1996; Núñez et al., 1998). Por otra parte, consideramos que sería oportuno mejorar y modificar los sistemas actuales de la enseñanza en Parasitología, al incorporar métodos avanzados de aprendizaje que van desde grupos de discusión, hasta la utilización de técnicas de enseñanza modernas, donde se puedan emplear, incluso, programas con soporte computadorizado (Arfaa, 1995; Theodoropoulus et al., 1997; Pawlowski et al., 1998).

\section{Agradecimientos}

Este proyecto no se hubiera desarrollado sin el apoyo financiero del Ministerio de Educación Superior del gobierno Flamenco de Bélgica. Le estamos muy agradecidos al profesor Paul H. de Rycke, promotor de la colaboración entre la Universidad de Gent, en Bélgica, y el Instituto "Pedro Kourî” de La Habana, Cuba; a los doctores Lázara Rojas, Humberto Marín, Vicente Montoto y Orlando Rojas; y a todos los profesores, por su importante aporte para el desarrollo de este trabajo en el país.

\section{Referencias}

ANDERSON, R. C., 1997. Some reflections on the teaching of Parasitology. Journal of Parasitology, 83:982-984.

ANONYMOUS, 1997. Outbreaks of pseudo-infection with Cyclospora and Cryptosporidium. MMWR, 46:354-358.

ARFAA, F., 1995. A computer-based book of medical helmintology. Computerized Methods and Programs in Biomedicine, 47:253-255.

CHAN, M. S., 1997. The global burden of intestinal nematode infections - Fifty years on. Parasitology Today, 13:438-443. 
ESCOBEDO, A. A. \& NUÑEZ, F. A., 1999. Prevalence of intestinal parasites in Cuban acquired immunodeficiency syndrome (AIDS) patients. Acta Tropica, 72:125-130.

EYCKMANS, L., 1997. Teaching tropical medicine: A personal view in perspective. Annals of Tropical Medicine \& Parasitology, 91:743-746.

FONTE, L.; MONTALVO, A. M.; ALBERTI, E.; NUÑEZ, F. \& ROJAS, L., 1998. Overdiagnosis of intestinal amoebiasis associated to serial microscopical examination of faeces. Some precisions on a problem. Memórias do Instituto Oswaldo Cruz, 93:799800.

GRIFFITHS, J. K., 1998. Human Cryptosporidiosis: Epidemiology, Transmission, Clinical Disease, Treatment, and Diagnosis. Advances in Parasitology, 40:37-85.

HADAD, P.; FERNANDEZ, H.; MILLAN, J. C.; RAMOS, A. \& NUÑEZ, F. A., 1993. Infección por Cryptosporidium sp. en individuos cubanos infectados por el VIH. Revista Cubana de Medicina Tropical, 45: 55-58.

HAQUE, R.; ALI, I. K. M.; AKTHER, S. \& PETRI, W. A., 1998. Comparison of PCR, Isoenzyme analysis, and antigen detection for diagnosis of Entamoeba histolytica infection. Journal of Clinical Microbiology, 36:449-452.

IAFAEV, R. K. H.; AVTUSHENKO, E. G.\& MEZHAZAKIS, F. I., 1998. Means for improving the teaching of Parasitology in higher medical academic institutions. Medicine and Parasitology, 1:47-49.

LAIRD, R.; RAMIREZ, E.; DEL RISCO, U.; CRESPO, F. M. \& DONA, M. C., 1996. Intervención para mejorar el diagnóstico coproparasitológico en la Provincia "Las Tunas", Cuba, 1993-1994. Boletín Chileno de Parasitología, 51:97-100.

MARSHALL, M. M.; NAUMOVITZ, D.; ORTEGA, Y. \& STERLING, C. R., 1997. Waterborne protozoan pathogens. Clinical Microbiology Reviews, 10:6785.

MORIN, C. A.; ROBERTS, C. L.; MSHAR, P. A.; ADISS, D. G. \& HADLER, J. L., 1997. What do physicians know about cryptosporidiosis? A survey of Connecticut physicians. Archives of Internal Medicine, 157:1017-1022.

NUÑEZ, F. A.; GALVEZ, M. D. \& FINLAY, C. M., 1995. Primer reporte en Cuba de infección intestinal humana por Cyclospora cayetanensis, Ortega, 1993. Revista Cubana de Medicina Tropical, 47:211-214.

NUÑEZ, F. A.; GINORIO, D.; CORDOVI, R. \& FINLAY, C. M., 1998. Intervención educativa para mejorar la calidad del diagnóstico coproparasitológico en la red de salud de Ciudad Habana, Cuba. Cadernos de Saúde Pública, 14:139-144.

NUÑEZ, F. A.; GINORIO, D. \& FINLAY, C. M., 1997. Control de la Calidad del Diagnóstico Coproparasitológico en la Provincia de Ciudad Habana, Cuba. Cadernos de Saúde Pública, 13:67-72.
NUÑEZ, F. A.; HERNANDEZ, M. \& FINLAY, C. M., 1996. A longitudinal study of Enterobiasis in three day care centers of Havana city. Revista do Instituto de Medicina Tropical de São Paulo, 38:129132.

NUÑEZ, F. A.; HERNANDEZ, M. \& FINLAY, C. M. 1999. Longitudinal study of giardiasis in three day care centres of Havana City. Acta Tropica, 73:237242.

NUÑEZ, F. A.; SANJURJO, E.; BRAVO, J. R.; CARBALLO, D. \& FINLAY, C. M., 1993. Trichuriasis en Cuba. Revista Cubana de Medicina Tropical, 45: 42-45.

ORIHEL, T. C.; ASH, L. R.; RAMACHANDRAN, C. P. \& OTTESEN, E., 1997. Medios Auxiliares para el Diagnóstico de las Parasitosis Intestinales. Geneva: Organización Mundial de la Salud.

ORTEGA, Y. R.; STERLING, C. R. \& GILMAN, R. H. 1998. Cyclospora cayetanensis. Advances in Parasitology, 40:399-418.

PAWLOWSKI, Z. S.; GOULLIER-FLEURET, A. \& BRUSCHI, F., 1998. Undergraduate teaching of Medical Parasitology. Parasitology Today, 14:127-128.

PETITHORY, J. C. \& DROUHET, E., 1990. Réalisations et perspective du contrùle de qualité en Parasitologie et Mycologie. Bulletin de la Société de Pathologie Exotique, 83:21-30.

RAMIREZ, E. \& DONA, M. C., 1993. Control de la Trichuriasis en una zona rural de Cuba. Parasitología al Día, 17:35-39.

SAVIOLI, L.; BUNDY, D. \& TOMKINS, A., 1992. Intestinal parasitic infections: A soluble public health problem. Transactions of the Royal Society of Tropical Medicine and Hygiene, 86:353-354.

SUAREZ, M.; DIAZ, M.; BUSTELO, J.; SANCHEZ, A. \& VIDAL, I., 1997. Cryptosporidiosis en niños con diarrea aguda de la provincia de Ciego de Avila, Cuba. Boletín Chileno de Parasitología, 52:50-54.

THEODOROPOULUS, G.; LOUMOS, V. \& TSOUROULAS, N., 1997. An Expert Parasite Identification (EPAION) system with multimedia support. Medicine Information of London, 22:263-273.

WEITZ, J. C. \& ASTORGA, B., 1995. Control de calidad externo en el diagnóstico parasitológico de Cryptosporidium parvum. Revista Médica de Chile, 123:997-1001.

WHO (World Health Organization), 1992. Informal consultation on intestinal protozoal infections. Mexico: WHO/Pan-American Health Organization.

WOOD, D. E.; PALMER, J.; MISSETT, P. \& WHITBY, J. L., 1994. Proficiency testing in Parasitology. An educational tool to improve laboratory performance. American Journal of Clinical Pathology, 102:393-394.

ZUEVA, L. O. \& LYSENKO, A., 1997. Current problems in the under- and postgraduate training of epidemiologists and parasitologists. Medicine and Parasitology, 3:3-34. 